\title{
Scarce Health-care Resources and Higher Case- fatality Rates Early in an Epidemic: Analysis of Hospitalized Patients with COVID-19 in Tianmen.
}

Jijia Hu

Wuhan University Renmin Hospital

Zongwei Zhang

Wuhan University Renmin Hospital

Wei Wang

The First People' Hospital of Tianmen

Yingang Zhang

The First People' Hospital of Tianmen

Juan Tian

The First People's Hospital of Tianmen

Ning Shao

The First People' Hospital of Tianmen

Nian Liu

The First People' Hospital of Tianmen

Zhihe Tao

The First People' Hospital of Tianmen

Hao Huang ( $\square$ ahuanghao@163.com )

The First People' Hospital of Tianmen https://orcid.org/0000-0003-1819-9292

Research

Keywords: COVID-19, SARS-CoV-2, Case-fatality rate, Health-care resource

Posted Date: April 16th, 2020

DOl: https://doi.org/10.21203/rs.3.rs-22958/v1

License: (c) (i) This work is licensed under a Creative Commons Attribution 4.0 International License.

Read Full License 


\section{Abstract}

\section{Background}

Tianmen had the highest case-fatality rate (CFR) among all the cities in China early in the transmission of SARS-CoV-2, but little is known about the details of the epidemic in Tianmen. Our study aims to reveal the causes of the high CFR from the aspects of clinical features, medical resources, and epidemic situation.

\section{Methods}

In this study, we report the admission and outcomes of 341 patients with confirmed COVID-19 who were admitted and hospitalized in the hospital (The First People's Hospital of Tianmen) from January 14 to February 25, 2020. Epidemic information and medical resources across different regions were collected and compared.

\section{Results}

All deaths from COVID-19 in Tianmen occurred in the hospital, and the proportion of patients with critical (8.5\%) symptoms in the hospital is higher than the average in China. In addition, the number of affected patients in Tianmen is quite low, but the hospital and the whole city had the highest CFR in the early stage of the epidemic. Completely different from the stable CFR in Hubei Province, the CFR of COVID-19 in the hospital and city dropped dramatically, from the largest value in China to a more average level, within a few days. Analysis of government public documents showed that the per capita medical resources in Tianmen are worse than those of Wuhan and Hubei.

\section{Conclusions}

Our findings suggest that the inadequate capacity to respond to public health emergencies caused by relatively scarce health-care resources is a crucial factor contributing to the higher CFR of infectious diseases in regions with lower economic levels. Such countries and territories should implement strategies earlier to minimize the danger of COVID-19.

\section{Background}

Coronavirus disease 2019 (COVID-19) had its first outbreak in Wuhan in December 2019 [1]. RT-PCR analyses of throat swab samples detected a novel coronavirus, named SARS-CoV-2 (previously known as 2019-nCoV). As of April 12, the number of cases of viral infection had surpassed 1696 588, and 105952 deaths have been reported globally [2]. As the epidemic continues to spread to many countries, COVID-19 demonstrates a clear human-to-human transmission characteristic and poses a severe threat to global health [3-4]. Therefore, the World Health Organization (WHO) declared COVID-19 a pandemic on March 12, 2020. As the first identified city of the pandemic, Wuhan (the capital city of Hubei Province) has been the subject of multiple studies that have analyzed the spread and pathogenicity of COVID-19 in the city 
[5-6]. However, less reports on the pandemic in other cities or towns in Hubei were documented up to date.

Tianmen is a medium-sized city in Hubei. Distinct from Wuhan, which has a population of more than 10 million, the number of residents in Tianmen was only 1.2 million in 2018. At present, COVID-19 has been effectively controlled in Tianmen. A total of 496 cases were diagnosed with COVID-19 and 13 deaths in Tianmen between January 14 and February 25, and no new infected patient appeared after February 25, all surviving patients were cured and discharged before March 21. Regretly, Tianmen had the highest CFR in China for a number of consecutive days early in the transmission of the virus. However, no study has investigated this phenomenon. In China, there is significant inequality in health-care resources among diverse regions; large, economically developed cities, for example, have abundant medical staff and equipment [7]. Therefore, the interregional and unequal capacity to respond to public health emergencies is evident. As of February 25, a total of 341 SARS-CoV-2-positive patients were admitted to our hospital (The First People's Hospital of Tianmen), the only designated center for the treatment of patients with severe or critical COVID-19 in Tianmen. During the clinical diagnosis and treatment of infected patients, we found that the situation of COVID-19 in Tianmen is slightly different from that in Wuhan and, indeed, all of Hubei. In this study, we aimed to find the possible causes of the high CFR in Tianmen and ultimately provide effective evidence for minimizing the danger of COVID-19.

\section{Methods}

\section{Study design and patients}

The research protocol was reviewed and approved by the Ethics Committee of The First People's Hospital of Tianmen, Hubei, China. The diagnosis and confirmation of COVID-19 was based on the New Coronavirus Pneumonia Prevention and Control Program of China (suspected symptoms, chest CT results and SARS-CoV-2 positivity by use of quantitative RT-PCR). The classification of COVID-19 was as follows: mild (absence of pneumonia on radiology); severe (fever, respiratory symptoms, pneumonia on radiography); critical (any of the following: 1 . shortness of breath, $\mathrm{RR} \geq 30$ per min, 2. oxygen saturation $\leq 93 \%$, or $3 . \mathrm{PaO}_{2} / \mathrm{FiO}_{2} \leq 300 \mathrm{mmHg}$ ). A total of 341 patients confirmed with COVID-19 were admitted from January 14 to February 25,2020 . Clinical charts and nursing records were reviewed to complete the record.

\section{Data Collection}

Admission information, classification, and outcomes of patients with COVID-19 were collected from the records. All public data were collected in documents from the Hubei Provincial Bureau of Statistics (http://tjj.hubei.gov.cn/) and Health Commission of Hubei Province (http://wjw.hubei.gov.cn/). Three researchers reviewed the data independently. 


\section{Statistical analysis}

Categorical variables are described with frequencies and percentages. Quantitative variables are described with the means (SDs) when normally distributed and with medians (IQRs) when non-normally distributed. Comparisons of quantitative variables between groups were performed using independentsample t-tests. Categorical variables between groups were expressed as numbers $(\mathrm{N}, \%)$ and compared with the $\chi^{2}$ test. A two-sided of $p$-value less than 0.05 was considered statistically significant. Statistical analyses were performed using SPSS software, version 21.0.

\section{Results}

\section{Baseline characteristics of patients with COVID-19}

As shown in Table 1, as of February 25, 341 confirmed COVID-19 patients had been hospitalized in our unit. The composition of the sexes and ages of these patients was similar to the data obtained from across China [8], which showed that males and older individuals are more likely to be infected and that patients with worse initial symptoms tend to be older. Most cases were classified as mild (79.2\%), and $12.3 \%$ of the cases were classified as severe COVID-19. In contrast, the proportion of critical (8.5\%) cases was higher than the average level in China [8]. In addition, the overall CFR was 3.8\%, and all deaths occurred in the critical group. Since the hospital is the only rescue center for patients with severe and critical COVID-19 in Tianmen, all deaths from the disease in this city occurred in the hospital and most died early in the outbreak (11 of 13 died before February 1 , supplementary Table 1 ). Therefore, a relatively high severity and fatality rate were observed in Tianmen in the early stages of the epidemic. Fortunately, most patients had a positive prognosis ( $40.8 \%$ cured and $52.5 \%$ improved). 
Table 1

Baseline characteristics of patients with COVID-19

\begin{tabular}{|c|c|c|c|c|}
\hline Characteristics & Overall & Mild & Severe & Critical \\
\hline Age, mean (IQR), y & $\begin{array}{l}46.13(35.00- \\
56.00)\end{array}$ & $\begin{array}{l}44.19(33.00- \\
55.00)\end{array}$ & $\begin{array}{l}51.79(43.75- \\
58.75)\end{array}$ & $\begin{array}{l}55.97(48.00- \\
65.00)\end{array}$ \\
\hline $\begin{array}{l}\text { Hospitalization day, } \\
\text { mean (SD), d }\end{array}$ & $16.70(6.17)$ & $16.85(5.78)$ & $22.05(6.54)$ & $8.07(5.96)$ \\
\hline \multicolumn{5}{|l|}{ Sex } \\
\hline Female, N (\%) & $147(43.1)$ & $123(45.6)$ & $13(31.0)$ & 11(37.9) \\
\hline Male, N (\%) & $194(56.9)$ & $147(54.4)$ & $29(69.0)$ & $18(62.1)$ \\
\hline \multicolumn{5}{|l|}{ Outcome } \\
\hline Cured, N (\%) & $139(40.8)$ & $115(42.6)$ & $22(52.4)$ & $2(6.9)$ \\
\hline Improved, N (\%) & $179(52.5)$ & $152(56.3)$ & $19(45.2)$ & $8(27.6)$ \\
\hline Unchanged, N (\%) & $10(2.9)$ & $3(1.1)$ & $1(2.4)$ & $6(20.7)$ \\
\hline Dead, N (\%) & $13(3.8)$ & 0 & 0 & $13(44.8)$ \\
\hline Total Number, N (\%) & $341(100)$ & $270(79.2)$ & $42(12.3)$ & $29(8.5)$ \\
\hline
\end{tabular}

\section{Marked Changes In The CFR In Tianmen}

To clarify the cause of the high CFR observed early in the transmission of the virus, we calculated and compared the CFR of patients with COVID-19 in the hospital and in all of Tianmen, Wuhan, and Hubei (Fig. 1). Since the start of the epidemic, the CFR across all of Hubei has been stable. However, confusingly, the CFR in the hospital and in all of Tianmen has changed greatly, starting at a high level in the early stages, dramatically declining from February 2 and then stabilizing after February 12. Specifically, until February 10, the CFR in Tianmen was higher than that in Wuhan and Hubei, but after February 12 it was lower than that of all of Hubei (Supplementary Table 1). This phenomenon has prompted us to pay closer attention to the early epidemic situation in Tianmen.

\section{Contradiction in the number of infections and the CFR in Tianmen}

To further determine the cause of the sharp changes in the CFR in Tianmen, we compared the number of infections per 10,000 population and the CFRs in different regions (Fig. 2). Since the CFR in Tianmen has stabilized since February 12, the number of infections and the CFRs of the three groups were collected and calculated from data obtained before February 12. Notably, the infection status and CFR are 
significantly different in diverse regions early in the outbreak (Fig. 2), Tianmen had the lowest average number of infections but the highest CFR (Supplementary Table 2). Considering that Tianmen's economy is relatively poor in the province, we propose that this condition may have been caused by insufficient medical resources.

\section{Comparison Of Health-care Resources}

Per capita public health-care resources are of great significance for the prevention and treatment of infectious diseases. To verify our hypothesis, we analyzed the medical resources in Tianmen, Wuhan and all of Hubei Province (Fig. 3 and Supplementary Table 3). As expected, the number of beds per thousand population in this city is much lower than in Wuhan and is even below the provincial average. In addition, in terms of the proportion of medical staff, the number of doctors and nurses per thousand people in Tianmen is also the lowest among these three groups. All evidence points to the relative shortage of medical resources in Tianmen when SARS-CoV-2 was beginning to spread as reasonably explaining the high CFR in the early stage of the epidemic.

\section{Discussion}

The outbreak of infectious disease COVID-19, caused by the novel coronavirus, has emerged as a global public health issue [1, 3]. Following the initial confirmation of COVID-19 in Wuhan, the capital of Hubei Province, SARS-CoV-2 has spread rapidly throughout numerous countries and territories worldwide. As the province with the most severe epidemic within China, Hubei had a relatively high CFR of COVID-19 [9]. Wuhan is a core city in China with a population of over 10 million, thus, the diagnosis and treatment of COVID-19 have been well reported and analyzed in the city. However, numerous small and medium-sized cities in Hubei are also facing a widespread SARS-CoV-2 infection.

Tianmen is one of the 17 cities in Hubei, located 169 kilometers from Wuhan, with a resident population of 1.2 million and one of the poorest cities in the province (GDP per capita \$ 6,588 in 2018, Total GDP ranking 16 of 17 in Hubei). Therefore, the spread of COVID-19 has truly brought trouble to this city. As of February 25, a total of 496 confirmed COVID-19 cases and 13 deaths have been reported in Tianmen. Our hospital is the only designated center of the treatment of severe and critical COVID-19 cases in the city, as of February 25, a total of 341 affected patients have been admitted to the hospital. The analysis we conducted in this manuscript revealed a larger proportion of critical cases and a higher CFR in the hospital compared with the average proportions and rates, respectively, across China. More unfortunately, compared to those of Wuhan and Hubei, in Tianmen the number of infections per 10,000 population was lower but the CFR was higher in the early stage. The situation in the hospital and this city truly reflected the contradiction between the lack of health-care resources and the sharp increase in the number of patients after the outbreak of COVID-19. To investigate the matter further, we analyzed government data and found that Tianmen's per capita medical staff and beds were much lower than those of Wuhan and Hubei, which reasonably indicated the heavier health-care burden early in the epidemic. However, with the 
strict implementation of patient isolation and the continuous support of external medical resources (more than 150 medical staff and a large amount of medical supplies from Shanxi Province), the CFR has gradually decreased. The abovementioned observations confirm the importance of reserve health-care resources for public health emergencies.

In the "Healthy China 2030 Framework", the Chinese government proposed that the purpose of reform is to promote equity and justice in the health field and gradually reduce differences in medical resources among regions [10]. Differences in health-care resources may directly affect the outcomes of public health emergencies. More importantly, COVID-19 now affects numerous countries and territories worldwide, including regions with relatively poor economic levels and limited medical resources, such as those in North Africa and the Middle East [11, 12]. More aggressive applications and preparations should be taken as soon as possible in such regions to limit or possibly reduce the effects of the epidemic.

\section{Conclusion}

This study illustrated the higher CFR early in an epidemic of COVID-19 in Tianmen, and also analyzed the difference in medical resources between regions with diverse economic levels, pointing out that high CFR may be related to the health-care resources shortage caused by the surge in patients. The uneven distribution of medical resources is quite common globally. With more and more regions facing the predicament of the spread of COVID-19, ways to achieve scientific health-care resource allocation and enhance the ability to respond to public health emergencies are issues that policymakers in China and even all countries through the world need to be addressed in the future. However, this study is only a single city study, and the reasons for the difference in COVID-19 outcomes between diverse regions or countries still need to be explored.

\section{Declarations}

\section{Author's contributions}

$\mathrm{HH}$ and $\mathrm{ZT}$ designed this study and played major roles in the clinical management of patients, data collection, and writing of the draft. ZZ and WW played major roles in the data collection and analysis. JH revised the data and finished the manuscript. YZ assisted in data collection. JT and NS played roles in the clinical management of patients. NL helped with the data collection and analysis. All authors reviewed and approved the final version of the manuscript.

\section{Competing interests}

All the authors declare no competing interests.

\section{Consent for publication}

Not applicable. 


\section{Ethics approval and consent to participate}

Not applicable.

\section{Availability of data and materials}

All data generated or used during the study are available in the submitted article.

\section{Acknowledgments}

Special thanks to my colleagues Qin Liu (Department of Medical Record Management, The First People's Hospital of Tianmen) and Zhou Yansheng (Information Center, The First People's Hospital of Tianmen) for their help in this study. Regret and blessings to those who have lost their loved ones in this terrible epidemic.

\section{Funding}

No funding supported this study.

\section{References}

1. Zhu N, Zhang D, Wang W, et al. A Novel Coronavirus from Patients with Pneumonia in China, 2019. New Engl J Med. 2020;382(8):727-33.

2. World Health Organization. Coronavirus disease 2019 (COVID-19) Situation report - 83, WHO. 2020. .

3. Wang C, Horby PW, Hayden FG, Gao GF. A novel coronavirus outbreak of global health concern. The Lancet. 2020;395(10223):470-3.

4. Huang $C$, Wang $Y$, Li X, et al. Clinical features of patients infected with 2019 novel coronavirus in Wuhan, China. The Lancet. 2020;395(10223):497-506.

5. Wang D, Hu B, Hu C, et al. Clinical Characteristics of 138 Hospitalized Patients With 2019 Novel Coronavirus-Infected Pneumonia in Wuhan, China. JAMA. 2020. doi:. [Online ahead of print].

6. Chen N, Zhou M, Dong X, et al. Epidemiological and clinical characteristics of 99 cases of 2019 novel coronavirus pneumonia in Wuhan, China: a descriptive study. The Lancet. 2020;395(10223):507-13.

7. Zhang T, Xu Y, Ren J, Sun L, Liu C. Inequality in the distribution of health resources and health services in China: hospitals versus primary care institutions. Int J Equity Health. 2017;16(1):42.

8. Wu Z, McGoogan JM. Characteristics of and Important Lessons from the Coronavirus Disease 2019 (COVID-19) Outbreak in China: Summary of a Report of 72314 Cases from the Chinese Center for Disease Control and Prevention. JAMA. 2020. doi:. [Online ahead of print].

9. Ji Y, Ma Z, Peppelenbosch MP, Pan Q. Potential association between COVID-19 mortality and healthcare resource availability. The Lancet Global Health. [Online ahead of print]. doi: .

10. Fu W, Zhao S, Zhang Y, Chai P, Goss J. Research in health policy making in China: out-of-pocket payments in Healthy China 2030. BMJ. 2018;360:k234. 
11. Makoni M. Africa prepares for coronavirus. The Lancet. 2020;395(10223):483.

12. Peeri NC, Shrestha N, Rahman MS, Zaki R, Tan Z, Bibi S, et al. The SARS, MERS and novel coronavirus (COVID-19) epidemics, the newest and biggest global health threats: what lessons have we learned? Int J Epidemiol. 2020. doi:. [Online ahead of print].

\section{Figures}

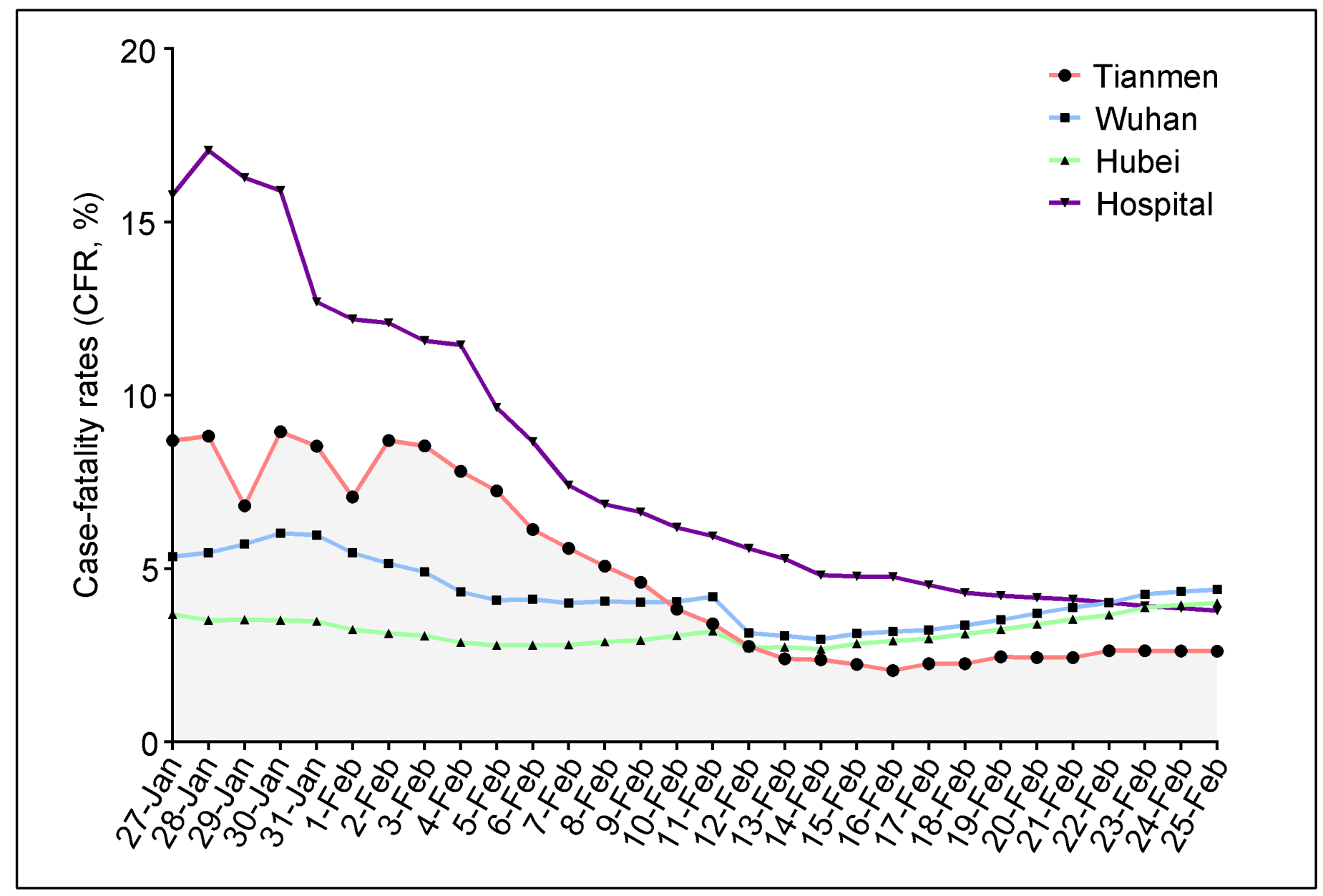

Figure 1

Comparison of case-fatality rates. We collected and calculated the CFRs of COVID-19 in The First People's Hospital of Tianmen (Hospital), Tianmen City (Tianmen), Wuhan City (Wuhan) and Hubei Province (Hubei) from January 27 to February 25. CFR, case-fatality rate. 


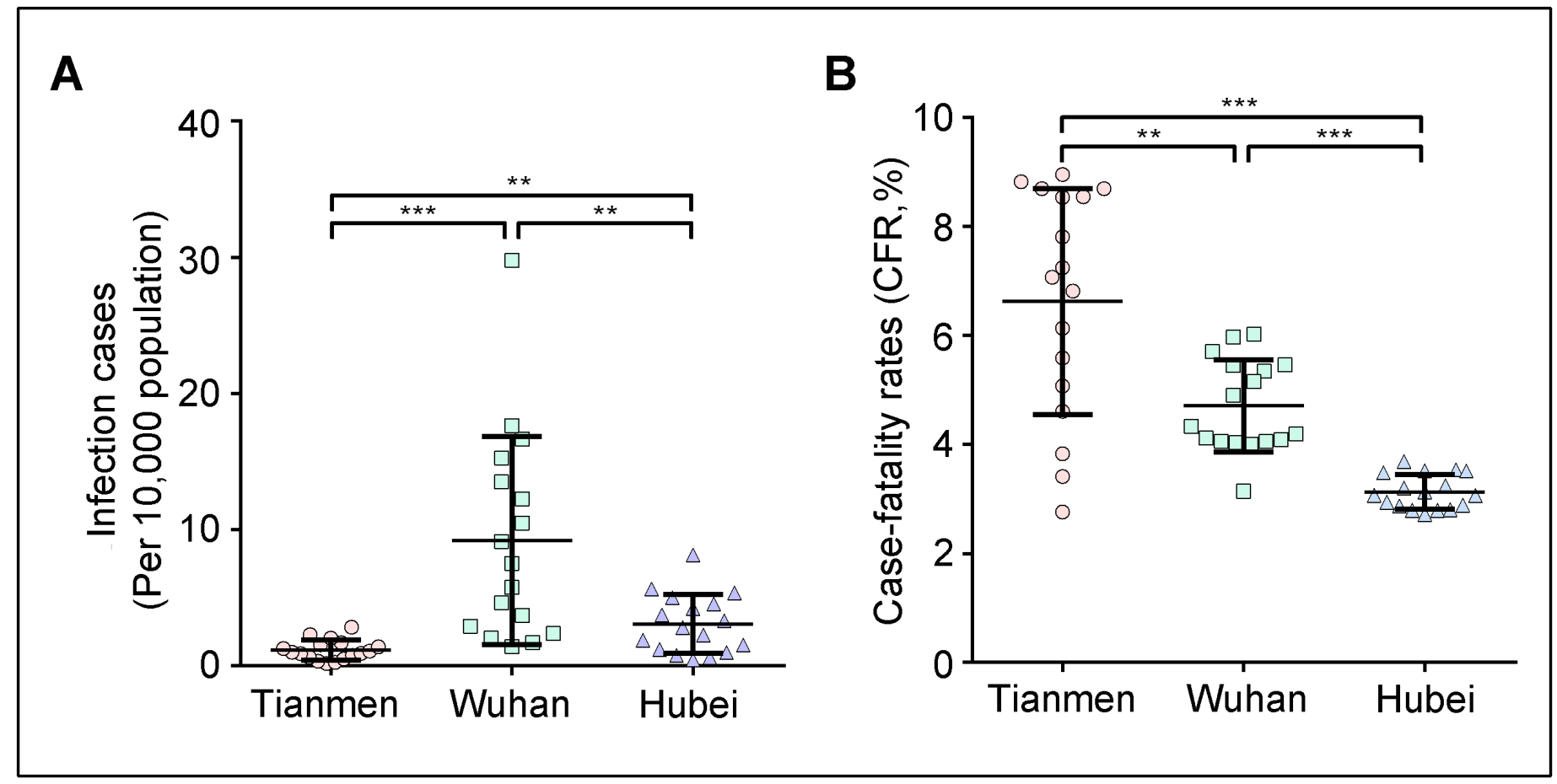

Figure 2

Comparison of the number of infections and the CFRs in different regions. The number of infections per 10,000 population and the CRFs of COVID-19 were calculated in Tianmen City (Tianmen), Wuhan City (Wuhan) and Hubei Province (Hubei) from January 27 to February 12. CFRs, case-fatality rates. ** $p<0.01$, $* \star \star ~ p<0.001$.

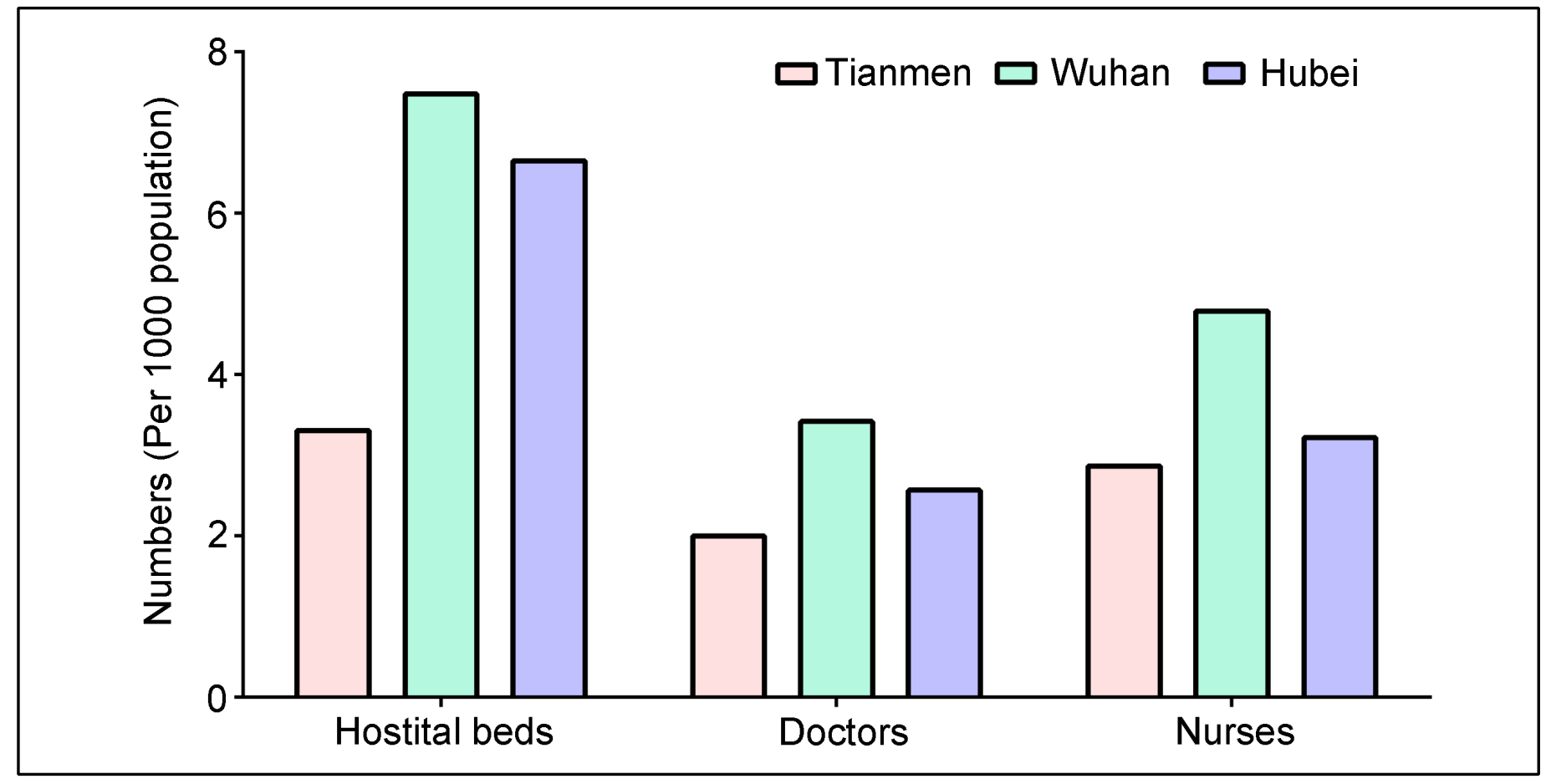

Figure 3 
Comparison of health-care resources in different regions. The number of beds in medical institutions, doctors and nurses per thousand population were collected in Tianmen City (Tianmen), Wuhan City (Wuhan) and Hubei Province (Hubei). Data as of the end of 2018.

\section{Supplementary Files}

This is a list of supplementary files associated with this preprint. Click to download.

- supple.docx 